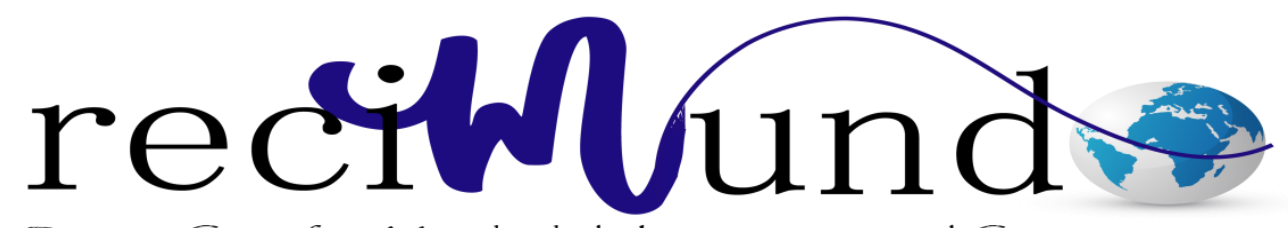

Revista Científica Mundo de la Investigación y el Conocimiento

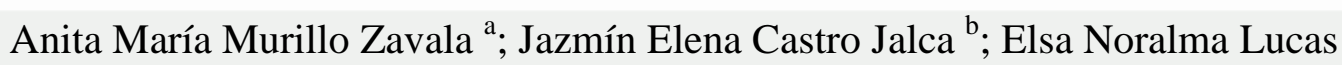
Parrales $^{c}$; Karina Maricela Merchán Villafuerte ${ }^{\text {d }}$

Infección parasitaria por Giardia lamblia en infantes

Revista Científica Mundo de la Investigación y el Conocimiento. Vol. 1 núm., 5, diciembre, 2017, pp. 860-870

DOI: $10.26820 /$ recimundo/1.5.2017.860-870

Editorial Saberes del Conocimiento

a. Universidad Estatal del Sur de Manabí; anita.murillo@unesum.edu.ec

b. Universidad Estatal del Sur de Manabí; jazmin.castro@unesum.edu.ec

c. Universidad Estatal del Sur de Manabí; elsa.lucas@unesum.edu.ec

d. Universidad Estatal del Sur de Manabí; karina.merchan@unesum.edu.ec 


\section{Infección parasitaria por Giardia lamblia en infantes}

Vol. 1, núm. 5., (2017)

Anita María Murillo Zavala; Jazmín Elena Castro Jalca; Elsa Noralma Lucas Parrales; Karina

Maricela Merchán Villafuerte

\section{RESUMEN}

La infección parasitaria causadas por el parasito Giardia intestinalis comúnmente destruye la mucosa del intestino delgado produciendo asi síndromes de mala absorción. Motivo por el cual se propuso realizar este estudio, cuyo objetivo principal fue determinar infección parasitaria por Giardia lamblia en niños de la Escuela Roberto Andrade, Recinto Chade- Cantón Jipijapa. La población que participó en ello fueron los niño/as que conforman el establecimiento, siendo un total de 32. Autorizando así participar en esta investigación los padres de familia como representantes de cada uno de ellos. Se utilizaron los métodos tales como analítico, descriptivo, estadístico y bibliográfico, para desarrollar y ejecutar el presente proyecto donde se conocieron las causas asociadas a las infecciones parasitarias por Giardia lamblia que afectan la salud de los niños entre ellos tenemos, que consumen agua sin hervir en un $44 \%$, procuran un lavado de manos al $16 \%$, consumen frutas o verduras sin lavar $12 \%$, carnes a medio coser $6 \%$, alimentos crudos 3\%, poseen animales cerca de los alimentos en un 19\%. Se observó, que tanto en el examen cuantitativo y cualitativo de Giardia lamblia hubo correlación entre estos dos métodos clínicos, encontrándose similitud de resultado en los casos positivos siendo un total de $38 \%$ en 12 estudiantes, los mismos que corresponden a 8 masculino y 4 femeninos, el $62 \%$ corresponde a resultados negativos siendo un total de 20 estudiantes incluyendo a 10 masculinos y 10 femeninos.

Palabras claves: Giardia, Infección parasitaria, antígeno, coproparasitario, diagnóstico clínico. 


\title{
Infección parasitaria por Giardia lamblia en infantes
}

Vol. 1, núm. 5., (2017)

Anita María Murillo Zavala; Jazmín Elena Castro Jalca; Elsa Noralma Lucas Parrales; Karina Maricela Merchán Villafuerte

\begin{abstract}
Parasitic infection caused by the parasite Giardia intestinalis commonly destroys the small intestine mucosa thus producing malabsorption syndromes. Why it was proposed this study, whose main objective was to determine parasite Giardia lamblia infection in children Roberto Andrade School Campus Chade- Jipijapa Canton. The people who participated in it were the child / as that comprise the establishment, with a total of 32 well Authorizing participate in this research parents as representatives of each of them. methods such as analytical and descriptive statistical and bibliographic were used to develop and implement this project where the causes associated with parasitic infections by Giardia lamblia affecting the health of children among them are, who consume water without met boil $44 \%$, handwashing seek to $16 \%$, consuming unwashed fruits or vegetables $12 \%$, meat medium sewing $6 \%, 3 \%$ raw foods, have animals near food by $19 \%$. It was observed that both the quantitative and qualitative examination of Giardia lamblia there was correlation between these two clinical methods, finding similarity of results in positive cases for a total of $38 \%$ to 12 students, the same as that of 8 male and 4 female, $62 \%$ are negative results for a total of 20 students including 10 male and 10 female.
\end{abstract}

Key words: Giardia, a parasitic infection, antigen, stools and clinical diagnosis. 


\section{Infección parasitaria por Giardia lamblia en infantes}

Vol. 1, núm. 5., (2017)

Anita María Murillo Zavala; Jazmín Elena Castro Jalca; Elsa Noralma Lucas Parrales; Karina

Maricela Merchán Villafuerte

\section{Introducción.}

La infección parasitaria por Giardia lamblia en la actualidad es un problema de salud frecuente en zonas rurales. Se propuso investigar sobre esta parasitosis en niños, población vulnerable de esta infección parasitaria considerando que en la actualidad los índices aumentan en países en vías de desarrollo donde influye la falta de servicios básicos, pobreza, desempleo, contaminación de los alimentos, agua potable no tratada y la inadecuada higiene personal razón por la cual surgió el interés de realizar la investigación.

La giardiasis, causada por Giardia lamblia, a nivel mundial tiene una distribución con mayor incidencia en regiones tropicales y subtropicales. Está demostrado que este parásito es el más frecuente en niños preescolares, persistiendo por más tiempo y con más intensidad que otros parásitos intestinales, causando efectos adversos tanto en el crecimiento, desarrollo como en el aprendizaje. ${ }^{1-3}$

Según el Md. Richard Pearson, manifiesta que las infecciones parasitarias son las responsables de una elevada tasa de morbimortalidad en todo el mundo, ya que estas infecciones son prevalentes en América Central y del Sur, África y Asia, y son menos frecuentes en Australia, Canadá, Europa, Japón, Nueva Zelanda y los Estados Unidos. Ya que su mayor impacto se da en residentes de países en vías de desarrollo, aunque también se dan en países desarrollados en migrantes y en viajeros que regresan de regiones endémicas. ${ }^{4}$

Según el informe de la Asociación de Médicos de Sanidad Exterior (AMSE) en Huelva España, manifiesta que a nivel mundial se ha estimado una frecuencia de 200'000.000 de individuos infectados por Giardia lamblia, de los cuales 500.000 sufren esta enfermedad. ${ }^{5}$ 


\section{Infección parasitaria por Giardia lamblia en infantes}

Vol. 1, núm. 5., (2017)

Anita María Murillo Zavala; Jazmín Elena Castro Jalca; Elsa Noralma Lucas Parrales; Karina Maricela Merchán Villafuerte

Según la Dra. Julia Collantes, médico de los laboratorios Pfizer en Quito manifiesta que en el Ecuador entre el $85 \%$ al $90 \%$ de la población sufre de parasitosis debido a las malas condiciones sanitarias y el mal uso de las letrinas. ${ }^{6}$

Los métodos utilizados en esta investigación fueron de gran utilidad ya que mostraron de manera detallada los hechos y objetivos que permitieron realizar este estudio, entre estos tenemos: Método Analítico permitió analizar mediante inmunocromatografía la detección cualitativa del antígeno de Giardia lamblia y demostró la presencia del parásito mediante examen coproparasitario, Método Descriptivo permitió dar a conocer las situaciones predominantes dentro de la investigación, Método Estadístico fue de gran utilidad ya que permitió resumir en tablas estadísticas los resultados a obtener, Método Bibliográfico permitió una amplia búsqueda de información sobre argumentos determinados mediante artículos científicos, revistas, editoriales y así fundamentar la investigación.

Mediante lo anteriormente enunciado se indagò sobre el tema propuesto, y se logró cumplir con los objetivos e hipótesis planteada los mismos que permitieron dar a conocer sobre infección parasitaria por Giardia lamblia.

El objetivo de la presente investigación es determinar infección parasitaria por Giardia lamblia en niños de la escuela Roberto Andrade, Recinto Chade- Cantón Jipijapa. 


\section{Infección parasitaria por Giardia lamblia en infantes}

Vol. 1, núm. 5., (2017)

Anita María Murillo Zavala; Jazmín Elena Castro Jalca; Elsa Noralma Lucas Parrales; Karina Maricela Merchán Villafuerte

\section{Metodología.}

\section{Población}

La población fue el total de los niños (as) de la Escuela Roberto Andrade. Recinto ChadeCantón Jipijapa.

Muestras

La muestra fue igual a la de la población por lo tanto se consideró a todos los niños (as) de la Escuela Roberto Andrade del Recinto Chade - Cantón Jipijapa siendo un total de 32.

\section{Métodos}

Para el desarrollo de la investigación se utilizaron los siguientes métodos:

\section{Método Analítico}

Este tipo de método permitió realizar un estudio del problema científico, y con ello se logró mediante la técnica de inmunocromatografía detectar de manera cualitativa el antígeno de Giardia lamblia, demostrando también la presencia del parásito mediante examen coproparasitario y comparar así los resultados obtenidos.

\section{Método Descriptivo}

Este tipo de método permitió dar a conocer las situaciones predominantes dentro de la investigación. 


\section{Infección parasitaria por Giardia lamblia en infantes}

Vol. 1, núm. 5., (2017)

Anita María Murillo Zavala; Jazmín Elena Castro Jalca; Elsa Noralma Lucas Parrales; Karina Maricela Merchán Villafuerte

\section{Método Estadístico}

Este método fue de gran utilidad ya que permitió resumir en tablas estadísticas los resultados a obtenerse sobre las infecciones parasitarias por Giardia lamblia.

\section{Método Bibliográfico}

Este método permitió una amplia búsqueda de información sobre argumentos determinados mediante artículos científicos, en libros de diferentes autores, editoriales, revistas y ediciones correspondientes, que dan respaldo a resultados investigativos acerca de las infecciones parasitarias por Giardia lamblia.

En la investigación se aplicarón todos los principios de bioética, incluyendo el consentimiento informado para los participantes involucrados en la misma.

Técnicas e Instrumentos

Las técnicas e instrumentos que se utilizaron fueron:

- Encuestas

- Entrevistas

- Bases de datos

- Registro de información

- Cámara fotográfica

\section{Resultados.}




\section{Infección parasitaria por Giardia lamblia en infantes}

Vol. 1, núm. 5., (2017)

Anita María Murillo Zavala; Jazmín Elena Castro Jalca; Elsa Noralma Lucas Parrales; Karina Maricela Merchán Villafuerte

Para llegar a alcanzar los objetivos planteados en esta investigación se aplicaron técnicas y métodos a lo largo de la misma.

La población que se estudió fueron 32 niños de la Escuela Roberto Andrade, Recinto Chade- Cantón Jipijapa, de las cuales el 44\% de género femenino y el 56\% de género masculino.

El objetivo específico establecido en la investigación fue identificar las causas asociadas a infecciones parasitarias por Giardia lamblia, se pudo cumplir empleando la técnica de encuesta a los padres de familia los mismo que se obtuvo como resultado que estos niño/as pueden llegar a contraer infecciones parasitarias, consumiendo agua sin hervir en un $44 \%$, no procuran el lavado de mano en un $16 \%$, consumen verduras o frutas sin cascaras sin lavar $12 \%$, consumen carnes a medio coser al $6 \%$, consumen alimentos crudos al $3 \%$, poseen animales cerca de los alimentos con un $19 \%$.

El segundo objetivo se pudo efectuar realizando la prueba del antígeno de Giardia lamblia la misma que se anticipó para su recolección y procesamiento, para obtener asi la presencia del antígeno.

El tercer objetivo se cumplió realizando de igual forma el examen coproparasitario lo que permitió confirmar la presencia del parásito.

En el cuarto objetivo se estableció una correlación entre el antígeno de Giardia lamblia y la presencia parasitaria por examen coproparasitario, en el cual tuvo similitud entre los porcentajes obtenidos ya que en los dos exámenes de laboratorio el 38\% corresponden a 


\section{Infección parasitaria por Giardia lamblia en infantes}

Vol. 1, núm. 5., (2017)

Anita María Murillo Zavala; Jazmín Elena Castro Jalca; Elsa Noralma Lucas Parrales; Karina Maricela Merchán Villafuerte

resultados positivos siendo un total de 12 pruebas, mientras que $62 \%$ corresponden a resultados negativos siendo un total de 20 pruebas.

Para comprobar la hipótesis planteada se afirmaba que el 65\% de los niños de la Escuela Roberto Andrade del Recinto Chade- Cantón Jipijapa presentaban infección parasitaria por Giardia lamblia, dando como rechazada esta hipótesis ya que la infección parasitaria está presente en un $38 \%$.

Dentro de las preguntas realizadas en la encuesta se fundamenta con lo siguiente:

- De acuerdo al lavado de las verduras y los vegetales los padres de familia contestaron que son directamente lavadas en el grifo con un $25 \%$, introduciendo en recipientes con un $75 \%$, y un $0 \%$ para otros.

- De acuerdo al proceder del agua de consumo, los padres de familia contestaron que el $44 \%$ consumen agua de pozo, vertiente $28 \%$, agua potable 16\%, bidón 12\%, $0 \%$ otros.

- Con respecto al lavado de manos de los niños al salir del baño/letrina contestaron los padres de familia en su totalidad que sus hijos si lavan sus manos en un 100\%, y un 0\% para un No.

- De acuerdo a que, si presentaban dolor abdominal sus niños, los padres de familia contestaron que si con un 44\%, mientras un 56\% no presentaban esta sintomatología.

- Con respecto a que si presentaban los niños al instante diarrea los padres contestaron que si dando un $25 \%$, mientras un $75 \%$ no presentaban diarrea. 


\section{Infección parasitaria por Giardia lamblia en infantes}

Vol. 1, núm. 5., (2017)

Anita María Murillo Zavala; Jazmín Elena Castro Jalca; Elsa Noralma Lucas Parrales; Karina Maricela Merchán Villafuerte

- Con respecto a que si habían tomado antiparasitarios hace días antes del examen contestaron que si dando un resultado del 31\%, mientras el $69 \%$ de los niños no habían ingerido ningún laxante.

- De acuerdo a que si tenían animales en sus domicilios, los padres de familia contestaron en su mayoría que poseen animales domésticos en un $69 \%$, mientras que $31 \%$ poseen animales de granja.

\section{Conclusiones.}

De acuerdo a las encuestas realizadas a los padres de familia se concluyó que no conocen en su totalidad acerca de las medidas de prevención ya que sus niños no cumplen con las respectivas normas de higiene y hábitos alimenticios para asi evitar estas infecciones.

En la investigación realizada se conoció que las infecciones parasitarias tienden a presentarse con mayor predisposición debido a la zona rural en la que habitan, la mayoría consumen carnes a medio coser, agua no tratada, frutas y verduras con cascaras sin lavar y no procuran correctamente el lavado de manos.

De acuerdo a los exámenes realizados a los niños se registraron que el 38\% fueron positivos para el antígeno de Giardia lamblia, mientras que el 62\% arrojaron resultados negativos, viéndose afectados en su mayoría los niños del género femenino.

Al realizar un análisis comparativo entre resultados en la búsqueda del antígeno de Giardia lamblia y los resultados de la búsqueda del parásito mediante el exámen coproparasitario 


\section{Infección parasitaria por Giardia lamblia en infantes}

Vol. 1, núm. 5., (2017)

Anita María Murillo Zavala; Jazmín Elena Castro Jalca; Elsa Noralma Lucas Parrales; Karina Maricela Merchán Villafuerte

se concluyó en que ambos dieron los mismos porcentajes tanto de casos positivos como de casos negativos.

Según la hipótesis planteada El 65\% de los niños de la Escuela Roberto Andrade del Recinto Chade- Cantón Jipijapa presentan infección parasitaria por Giardia lamblia, por lo que la hipótesis fue rechazada ya que la infección parasitaria estuvo presente en el 38\% de los niños.

\section{Bibliografía.}

1. Giraldo-Gómez JM, Lora F, Henao LH, Mejía S. Prevalencia de Giardiasis y Parásitos Intestinales en Preescolares de Hogares atendidos en un programa estatal en Armenia, Colombia. Rev. salud pública. 2005 Ene; 7(3): p. 327-338.

2. Luján HD. Giardia y giardiasis. Medicina (Buenos Aires). 2006 Ene; 66(1): p. 70-74.

3. Rivera M, De la Parte MA, Hurtado P, Magaldi L. Giardiasis intestinal. Mini-Revisión. Investigación Clínica. 2002 Abr; 43(2): p. 13-19.

4. Pearson RD. Abordaje de las infecciones parasitarias Kenilworth: MSD; 2010.

5. AMSE. TUBERCULOSIS: Epidemiología y Situación mundial Huelva: ASOCIACIÓN DE MÉDICOS DE SANIDAD EXTERIOR; 2012.

6. Collantes J. LA HORA NACIONAL. Los parasitos afectan al 90\% de los Ecuatorianos. 2011 Julio 9. 